Commun. math. Phys. 31, 161-170 (1973)

(C) by Springer-Verlag 1973

\title{
The Four Laws of Black Hole Mechanics
}

\author{
J. M. Bardeen $\star$ \\ Department of Physics, Yale University, New Haven, Connecticut, USA \\ B. Carter and S. W. Hawking \\ Institute of Astronomy. University of Cambridge, England
}

Received January 24, 1973

\begin{abstract}
Expressions are derived for the mass of a stationary axisymmetric solution of the Einstein equations containing a black hole surrounded by matter and for the difference in mass between two neighboring such solutions. Two of the quantities which appear in these expressions, namely the area $\mathrm{A}$ of the event horizon and the "surface gravity" $\kappa$ of the black hole, have a close analogy with entropy and temperature respectively. This analogy suggests the formulation of four laws of black hole mechanics which correspond to and in some ways transcend the four laws of thermodynamics.
\end{abstract}

\section{Introduction}

It is generally believed that a gravitationally collapsing body will give rise to a black hole and that this black hole will settle down to a stationary state. If the black hole is rotating, the stationary state must be axisymmetric [1] (An improved version of this theorem involving weaker assumptions is outlined in [2] and is given in detail in [3]). It has been shown that stationary axisymmetric black hole solutions which are empty outside the event horizon fall into discrete families each of which depends on only two parameters, the mass $M$ and the angular momentum $J$ [4-6]. The Kerr solutions for $M^{4}>J^{2}$ are one such family. It seems unlikely that there are any others. It also seems reasonable to suppose that the Newman-Kerr solutions for $M^{4}>J^{2}+M^{2} Q^{2}$, where $Q$ is the electric charge, are the only stationary axisymmetric black hole solutions which are empty outside the event horizon apart from an electromagnetic field. On the other hand there will be an infinite dimensional family of stationary axisymmetric solutions in which there are rings of matter orbiting the black hole. In Sections 2 and 3 of this paper we shall derive formulae for the mass of such a solution and for the difference in mass of two nearby solutions. These formulae

\footnotetext{
* Research supported in part by the National Science Foundation.
} 
generalise the expressions found by Smarr [7] and Beckenstein [8] for the Kerr and Newman-Kerr solutions. We show that the quantities appearing in the formulae have well-defined physical interpretations. Of particular interest are the area $A$ of the event horizon and the "surface gravity" $\kappa$, which appear together. These have strong analogies to entropy and temperature respectively. Pursuing this analogy we are led in Section 4 to formulate four laws of black hole mechanics which are similar to, but distinct from, the four laws of thermodynamics.

\section{The Integral Formula}

In a stationary axisymmetric asymptotically flat space, there is a unique time translational Killing vector $K^{a}$ which is timelike near infinity with $K^{a} K_{a}=-1$ and a unique rotational Killing vector $\tilde{K}^{a}$ whose orbits are closed curves with parameter length $2 \pi$. These Killing vectors obey equations

$$
\begin{aligned}
K_{a ; b} & =K_{[a ; b]}, \quad \tilde{K}_{a ; b}=\tilde{K}_{[a ; b]}, \\
K_{a ; b} \tilde{K}^{b} & =\tilde{K}_{a ; b} K^{b}, \\
K^{a ; b}{ }_{b} & =-R^{a}{ }_{b} K^{b}, \\
\tilde{K}^{a ; b}{ }_{b} & =-R^{a}{ }_{b} \tilde{K}^{b},
\end{aligned}
$$

where a semicolon denotes the covariant derivatives, square brackets around indices imply antisymmetrization and $R_{a b}=R_{a c b}{ }^{c}$ with

$$
v_{d ;[b c]}=\frac{1}{2} R_{a d b c} v^{a}
$$

for any vector $v^{a}$. Since $K_{a ; b}$ is antisymmetric, one can integrate Eq. (3) over a hypersurface $S$ and transfer the volume on the left to an integral over a 2 -surface $\partial S$ bounding $S$ :

$$
\int_{i S} K^{a ; b} d \Sigma_{a b}=-\int_{S} R_{b}^{a} K^{b} d \Sigma_{a},
$$

where $d \Sigma_{a b}$ and $d \Sigma_{a}$ are the surface elements of $\partial S$ and $S$ respectively. We shall choose the surface to be spacelike, asymptotically flat, tangent to the rotation Killing vector $\tilde{K}^{a}$, and to intersect the event horizon [1] in a 2-surface $\partial B$. The boundary $\partial S$ of $S$ consists of $\partial B$ and a 2-surface $\partial S_{\infty}$ at infinity. For an asymptotically flat space, the integral over $\partial S_{\infty}$ in equation (5) is equal to $-4 \pi M$, where $M$ is the mass as measured from infinity. Thus

where

$$
M=\int_{S}\left(2 T_{a}^{b}-T \delta_{a}^{b}\right) K^{a} d \Sigma_{b}+\frac{1}{4 \pi} \int_{\partial B} K^{a ; b} d \Sigma_{a b},
$$

$$
R_{a b}-\frac{1}{2} R g_{a b}=8 \pi T_{a b} \text {. }
$$


The first integral on the right can be regarded as the contribution to the total mass of the matter outside the event horizon, and the second integral may be regarded as the mass of the black hole. One can integrate Eq. (4) similarly to obtain an expression for the total angular momentum $J$ as measured asymptotically from infinity,

$$
J=-\int_{S} T_{b}^{a} \tilde{K}^{b} d \Sigma_{a}-\frac{1}{8 \pi} \int_{\partial B} \tilde{K}^{a ; b} d \Sigma_{a b} .
$$

The first integral on the right is the angular momentum of the matter, and the second integral can be regarded as the angular momentum of the black hole.

One can introduce a time coordinate $t$ which measures the parameter distance from $S$ along the integral curves of $K^{a}$ (i.e. $t_{; a} K^{a}=1$ ). The null vector $l^{a}=d x^{a} / d t$, tangent to the generators of the horizon, can be expressed as

$$
l^{a}=K^{a}+\Omega_{H} \tilde{K}^{a} .
$$

The coefficient $\Omega_{H}$ is the angular velocity of the black hole and is the same at all points of the horizon [9]. Thus one can rewrite Eq. (6) as

where

$$
M=\int_{S}\left(2 T_{a}^{b}-T \delta_{a}^{b}\right) K^{a} d \Sigma_{b}+2 \Omega_{H} J_{H}+\frac{1}{4 \pi} \int_{\partial B} l^{a ; b} d \Sigma_{a b},
$$

$$
J_{I I}=-\frac{1}{8 \pi} \int_{\partial B} \tilde{K}^{a ; b} d \Sigma_{a b}
$$

is the angular momentum of the black hole. One can express $d \Sigma_{a b}$ as $l_{[a} n_{b]} d A$, where $n_{a}$ is the other null vector orthogonal to $\partial B$, normalized so that $n_{a} l^{a}=-1$, and $d A$ is the surface area element of $\partial B$. Thus the last term on the right of Eq. (9) is

$$
\frac{1}{4 \pi} \int_{\partial B} \kappa d A
$$

where $\kappa=-l_{a ; b} n^{a} l^{b}$ represents the extent to which the time coordinate $t$ is not an affine parameter along the generators of the horizon. One can think of $\kappa$ as the "surface gravity" of the black hole in the following sense: a particle outside the horizon which rigidly corotates with the black hole has an angular velocity $\Omega_{H}$, a four-velocity $v^{a}=v^{t}\left(K^{a}+\Omega_{H} \tilde{K}^{a}\right)$, and an acceleration four-vector $v_{; b}^{a} v^{b}$. The magnitude of the acceleration, multiplied by a factor $1 / v^{t}$ to convert from change in velocity per unit proper time to change in velocity per unit coordinate time $t$, tends to $\kappa$ when the particle is infinitesimally close to the event horizon.

We shall now show that $\kappa$ is constant over the horizon. Let $m^{a}, \bar{m}^{a}$ be complex conjugate null vectors lying in $\partial B$ and normalised so that 
$m^{a} \bar{m}_{a}=1$. Then

$$
\begin{aligned}
\kappa_{; a} m^{a} & =-\left(l_{a ; b} n^{a} l^{b}\right)_{; c} m^{c} \\
& =-l_{a ; b c} n^{a} l^{b} m^{c}-l_{a ; b} n_{; c}^{a} l^{b} m^{c}-l_{a ; b} n^{a} l_{; c}^{b} m^{c} .
\end{aligned}
$$

Since $l^{a}$ is a Killing vector, $l_{a ; b c}=R_{d c b a} l^{d}$. The normalization of the null tetrad on the horizon, from which

$$
g_{a b}=-n_{a} l_{b}-l_{a} n_{b}+m_{a} \bar{m}_{b}+\bar{m}_{a} m_{b},
$$

is used to put the second term in the form $\kappa l_{a ; c} n^{a} m^{c}$. The third term is $-\kappa l_{a ; c} n^{a} m^{c}$ as a result of the vanishing of the shear and convergence of the generators of the horizon, $l_{a ; b} m^{a} \bar{m}^{b}=0=l_{a ; b} m^{a} m^{b}$. Thus

$$
\kappa_{; a} m^{a}=-R_{a b c d} l^{a} m^{b} l^{c} n^{d} .
$$

But on the horizon

$$
\begin{aligned}
0 & =\left(l_{a ; b} m^{a} \bar{m}^{b}\right)_{; c} m^{c} \\
& =R_{d a b c} l^{d} m^{a} \bar{m}^{b} m^{c} \\
& =-R_{d b} l^{d} m^{b}+R_{a b c d} l^{a} m^{b} l^{c} n^{d} .
\end{aligned}
$$

By the Einstein equations $R_{b d} l^{b} m^{d}=8 \pi T_{b d} l^{b} m^{d}$.

If energy-momentum tensor obeys the Dominant Energy Condition [10], $T_{b d} l^{b}$ will be a non-spacelike vector. However $T_{b d} l^{b} l^{d}=0$ on the horizon since the shear and convergence of the horizon are zero. This shows that $T_{b d} l^{b}$ must be zero or parallel to $l^{d}$ and that $T_{b d} l^{b} m^{d}=0$. Thus $\kappa_{; a} m^{a}$ is zero and $\kappa$ is constant on the horizon.

The integral mass formula becomes

$$
M=\int_{S}\left(2 T_{a}^{b}-T \delta_{a}^{b}\right) K^{a} d \Sigma_{b}+2 \Omega_{H} J_{H}+\frac{\kappa}{4 \pi} A,
$$

where $A$ is the area of a 2-dimensional cross section of the horizon. When $T_{a b}$ is zero, i.e. when the space outside the horizon is empty, this formula reduces to that found by Smarr [7] for the Kerr solution. In the Kerr solution,

$$
\begin{gathered}
\Omega_{H}=\frac{J_{H}}{2 M\left(M^{2}+\left(M^{4}-J_{H}^{2}\right)^{1 / 2}\right)}, \\
\kappa=\frac{\left(M^{4}-J_{H}^{2}\right)^{1 / 2}}{2 M\left(M^{2}+\left(M^{4}-J_{H}^{2}\right)^{1 / 2}\right)}, \\
A=8 \pi\left(M^{2}+\left(M^{4}-J_{H}^{2}\right)^{1 / 2}\right) .
\end{gathered}
$$

For a Kerr solution with a zero angular momentum, the total mass is represented by the last term in equation (13). As the angular momentum increases, the surface gravity decreases until it is zero in the limiting case, $J_{H}^{2}=M^{4}$. The mass is then all represented by the rotational term 
$2 \Omega_{H} J_{H}$. The reduction of the surface gravity with angular momentum can be thought of as a centrifugal effect. When the angular momentum is near the limiting value, the horizon is, in a sense, very loosely bound and a small perturbation can raise a large tide [11].

\section{The Differential Formula}

In this section we shall use the integral mass formula to derive an expression for the difference $\delta M$ between the masses of two slightly different stationary axisymmetric black hole solutions. For simplicity we shall consider only the case in which the matter outside the horizon is a perfect fluid in circular orbit around the black hole. The differential mass formula for rotating stars without the blackhole terms is discussed in [12]. A treatment including electromagnetic fields, which allows the matter to be an elastic solid, is given in [6].

A perfect fluid may be described by an energy density $\varepsilon$ which is a function of the particle number density $n$ and entropy density $s$. The temperature $\theta$, chemical potential $\mu$ and pressure $p$ are defined by

$$
\begin{aligned}
& \theta=\frac{\partial \varepsilon}{\partial s}, \\
& \mu=\frac{\partial \varepsilon}{\partial n}, \\
& p=\mu n+\theta s-\varepsilon .
\end{aligned}
$$

The energy momentum tensor is

$$
T_{a b}=(\varepsilon+p) v_{a} v_{b}+p g_{a b},
$$

where $v^{a}=\left(-u_{b} u^{b}\right)^{-1 / 2} u^{a}$ is the unit vector tangent to the flow lines and $u^{a}=K^{a}+\Omega \tilde{K}^{a}$, where $\Omega$ is the angular velocity of the fluid. The angular momentum, entropy and number of particles of the fluid can be expressed as

and

$$
\begin{gathered}
-\int T^{a}{ }_{b} \tilde{K}^{b} d \Sigma_{a}, \\
\int s v^{a} d \Sigma_{a},
\end{gathered}
$$

$$
\int n v^{a} d \Sigma_{a} \text { respectively . }
$$

When comparing two slightly different solutions there is a certain freedom in which points are chosen to correspond. We shall use this freedom to make the surfaces $S$, the event horizons, and the Killing vectors $K^{a}$ and $\tilde{K}^{a}$ the same in the two solutions. Thus

and

$$
\delta K^{a}=\delta \tilde{K}^{a}=0
$$

$$
\delta K_{a}=h_{a b} K^{b}, \quad \delta \tilde{K}_{a}=h_{a b} \tilde{K}^{b},
$$


where $h_{a b}=\delta g_{a b}=-g_{a c} g_{b d} \delta g^{c d}$. Then

$$
\begin{aligned}
& \delta l^{a}=\delta \Omega_{H} \tilde{K}^{a}, \\
& \delta l_{a}=h_{a b} l^{b}+g_{a b} \delta \Omega_{H} \tilde{K}^{b} .
\end{aligned}
$$

Since the event horizons are in the same position in the two solutions, the covariant vectors normal to them must be parallel,

$$
\delta l_{[a} l_{b]}=0, \quad \delta n^{[a} n^{b]}=0 .
$$

Also, the Lie derivative of $\delta l_{a}$ by $l^{b}$ is zero, $\left(\delta l_{a}\right)_{; b} l^{b}+\delta l_{a} l_{; b}^{a}=0$. Therefore

$$
\begin{aligned}
\delta \kappa= & \frac{1}{2}\left(\delta l_{a} l^{a}+l_{a} \delta l^{a}\right)_{; c} n^{c}+\frac{1}{2}\left(l_{a} l^{a}\right)_{; c} \delta n^{c} \\
= & \frac{1}{2}\left(\delta l_{a}\right)_{; b}\left(l^{a} n^{b}+n^{a} l^{b}\right)+\delta l_{a} l_{; b}^{a} n^{b} \\
& +\delta \Omega_{H} \tilde{K}^{a}{ }_{;} l_{a} n^{b}+\delta n^{b} l_{; b}^{a} l_{a} \\
= & \frac{1}{2}\left(\delta l_{a}\right)_{; b}\left(l^{a} n^{b}+n^{a} l^{b}\right)+\delta \Omega_{H} \tilde{K}_{; b}^{a} l_{a} n^{b} .
\end{aligned}
$$

As $\delta l_{a}$ is proportional to $l_{a}$ on the horizon, $\left(\delta l_{a}\right)_{; b} m^{a} \bar{m}^{b}$ is zero. Thus

$$
\begin{aligned}
\delta \kappa & =-\frac{1}{2}\left(\delta l_{a}\right)^{; a}+\delta \Omega_{H} \tilde{K}^{a}{ }_{b} l_{a} n^{b} \\
& =-\frac{1}{2} h_{a b}{ }^{; a} l^{b}+\delta \Omega_{H} \tilde{K}_{; b}^{a} l_{a} n^{b} .
\end{aligned}
$$

To evaluate $\delta M$, we express the mass formula derived in the previous section in the form

$$
M=\int_{S}\left(2 T_{a}^{b}+\frac{1}{8 \pi} R \delta_{a}^{b}\right) K^{a} d \Sigma_{b}+2 \Omega_{H} J_{H}+\frac{\kappa}{4 \pi} A .
$$

The variation of the term involving the scalar curvature, $R$, gives

But

$$
-\frac{1}{8 \pi} \int_{S}\left\{\left(R_{c d}-\frac{1}{2} g_{c d} R\right) h^{c d}+2 h_{[c ; d]}^{c ; d}\right\} K^{a} d \Sigma_{a} .
$$

$$
2 h_{[c ; d]}^{c ; d} K^{a}=2\left(K^{a} h_{c}^{[c ; d]}-K^{d} h_{c}^{[c ; a]}\right)_{; d},
$$

using $h_{c d ; a} K^{a}+h_{a d} K^{a}{ }_{; c}+h_{a c} K^{a} ; d=0$. One can therefore transform the last term in (29) into the 2-surface integral

$$
-\frac{1}{4 \pi} \int_{\partial S}\left(K^{a} h_{c}^{[c ; d]}-K^{d} h_{c}^{[c ; a]}\right) d \Sigma_{a d} .
$$

The integral over $\partial S_{\infty}$ gives $-\delta M$ and, by Eq. (27), the integral over $\partial B$ gives $-\frac{\delta \kappa}{4 \pi} A-2 \delta \Omega_{H} J_{H}$.

The variation of the energy-momentum tensor term in (28) is

$$
\begin{aligned}
2 \delta \int T_{a}^{b} K^{a} d \Sigma_{b}= & -2 \int \Omega \delta\left\{T_{a}^{b} \tilde{K}^{a} d \Sigma_{b}\right\}+2 \delta \int p K^{a} d \Sigma_{a} \\
& +2 \int u^{a} \delta\left\{(\varepsilon+p)\left(-u^{c} u^{d} g_{c d}\right)^{-1} u_{a} K^{b} d \Sigma_{b}\right\} .
\end{aligned}
$$


But $\varepsilon+p=\mu n+\theta s, \delta p=\delta \mu n+\delta \theta s$, and $u^{a} \delta\left\{\left(-u^{c} u^{d} g_{c d}\right)^{-1 / 2} u_{a}\right\}=\frac{1}{2} v^{c} v^{d} h_{c d}$. Therefore

$$
\begin{aligned}
2 \delta \int T_{a}^{b} K^{a} d \Sigma_{b}= & \int T^{c d} h_{c d} K^{a} d \Sigma_{a}+2 \int \Omega \delta d J \\
& +2 \int \bar{\mu} \delta d N+2 \int \bar{\theta} \delta d S,
\end{aligned}
$$

where $\delta d J=-\delta\left\{T_{a}^{b} \tilde{K}^{a} d \Sigma_{b}\right\}$ is the change in the angular momentum of the fluid crossing the surface element $d \Sigma_{b}$,

$$
\delta d N=\delta\left\{n\left(-u_{a} u^{a}\right)^{-1 / 2} K^{b} d \Sigma_{b}\right\}
$$

is the change in the number of particles crossing $d \Sigma_{b}$,

$$
\delta d S=\delta\left\{s\left(-u_{a} u^{a}\right)^{-1 / 2} K^{b} d \Sigma_{b}\right\}
$$

is the change in the entropy crossing $d \Sigma_{b}$,

$$
\bar{\mu}=\left(-u_{a} u^{a}\right)^{1 / 2} \mu
$$

is the "red-shifted" chemical potential, and

$$
\bar{\theta}=\left(-u_{a} u^{a}\right)^{1 / 2} \theta
$$

is the "red-shifted" temperature. Thus

$$
\delta M=\int \Omega \delta d J+\int \bar{\mu} \delta d N+\int \bar{\theta} \delta d S+\Omega_{H} \delta J_{H}+\frac{\kappa}{8 \pi} \delta A .
$$

This is the differential mass formula.

If an infinitesimal ring is added to a black hole slowly, without allowing any matter or radiation to cross the event horizon, the area and the angular momentum of the black hole are constant and the matter terms in the Eq. (34) give the net energy required to add the ring. Since $\Omega_{H}$ and $\kappa$ do change to first order in the mass of the ring, the change in $M_{H}=2 \Omega_{H} J_{H}+\kappa A / 4 \pi$ must be taken into account in the integral mass formula of Eq. (13).

\section{The Four Laws}

In this section we shall pursue the analogy between black holes and thermodynamics and shall formulate four laws which correspond to and in some ways transcend the four laws of thermodynamics. We start with the most obvious analogy:

\section{The Second Law [1]}

The area $A$ of the event horizon of each black hole does not decrease with time, i.e.

$$
\delta A \geqq 0 \text {. }
$$


If two black holes coalesce, the area of the final event horizon is greater than the sum of the areas of the initial horizons, i.e.

$$
A_{3}>A_{1}+A_{2} \text {. }
$$

This establishes the analogy between the area of the event horizon and entropy. The second law of black hole mechanics is slightly stronger than the corresponding thermodynamic law. In thermodynamics one can transfer entropy from one system to another, and it is required only that the total entropy does not decrease. However one cannot transfer area from one black hole to another since black holes cannot bifurcate $([1,2,3])$. Thus the second law of black hole mechanics requires that the area of each individual black hole should not decrease.

\section{The First Law}

Any two neighboring stationary axisymmetric solutions containing a perfect fluid with circular flow and a central black hole are related by

$$
\delta M=\frac{\kappa}{8 \pi} \delta A+\Omega_{H} \delta J_{H}+\int \Omega \delta d J+\int \bar{\mu} \delta d N+\int \bar{\theta} \delta d S .
$$

It can be seen that $\frac{\kappa}{8 \pi}$ is analogous to temperature in the same way that $A$ is analogous to entropy. It should however be emphasized that $\frac{\kappa}{8 \pi}$ and $A$ are distinct from the temperature and entropy of the black hole.

In fact the effective temperature of a black hole is absolute zero. One way of seeing this is to note that a black hole cannot be in equilibrium with black body radiation at any non-zero temperature, because no radiation could be emitted from the hole whereas some radiation would always cross the horizon into the black hole. If the wavelength of the radiation were very long, corresponding to a low black body temperature, the rate of absorption of radiation would be very slow, but true equilibrium would be possible only if there were no radiation present at all, i.e. if the external black body radiation temperature were zero. Another way of seeing that the effective temperature of a black hole is zero is to note that the "red shifted" effective temperature $\bar{\theta}$ of any matter orbiting the black hole must tend to zero as the horizon is approached, because the time dilatation factor $\left(-u^{a} u_{a}\right)^{1 / 2}$ tends to zero on the horizon. The fact that the effective temperature of a black hole is zero means that one can in principle add entropy to a black hole without changing it in any way. In this sense a black hole can be said to transcend the 
second law of thermodynamics. In practise of course any addition of entropy to a black hole would cause some increase in the area of the event horizon. One might therefore suppose that by adding some multiple of the area to the total entropy of all matter outside the event horizon one could obtain a quantity which never decreased. However this is not possible since by careful management one can arrange that the area increase accompanying a given addition of entropy is arbitrarily small. One way of doing this would be to put the entropy into two containers and lower them on ropes down the axis towards the north and south poles. As the containers approach the black hole they would distort the horizon. The shear or rate of distortion of the horizon would be proportional to the rate at which the containers were being lowered. The rate of increase of area of the horizon would be proportional to the square of the shear, $[2,11]$, and so to the square of the rate at which the containers were being lowered. Thus by lowering the containers very slowly, one could ensure that the area increase was very small. When the containers reach the horizon, they would be moving parallel to the null vector $l^{a}$ and so would not cause any area increase as they cross the horizon.

In a similar way the effective chemical potential $\bar{\mu}$ tends to zero on the horizon, which means that in principle one can also add particles to a black hole without changing it. In this sense a black hole transcends the law of conservation of baryons.

Continuing the analogy between $\frac{\kappa}{8 \pi}$ and temperature, one has:

\section{The Zeroth Law}

The surface gravity, $\kappa$ of a stationary black hole is constant over the event horizon.

This was proved in Section 2. Other proofs under slightly different assumptions are given in $[6,2]$.

Extending the analogy even further one would postulate:

\section{The Third Law}

It is impossible by any procedure, no matter how idealized, to reduce $\kappa$ to zero by a finite sequence of operations.

This law has a rather different status from the others, in that it does not, so far at least, have a rigorous mathematical proof. However there are strong reasons for believing in it. For example if one tries to reduce the value of $\kappa$ of a Kerr black hole by throwing in particles to increase the angular momentum, one finds that the decrease in $\kappa$ per particle thrown in gets smaller and smaller as the mass and angular momentum 
tend to the critical ratio $J / M^{2}=1$ for which $\kappa$ is zero. While idealized accretion processes do exist for which $J / M^{2} \rightarrow 1$ with the addition of a finite amount of rest mass $([13,14])$, they require an infinite divisibility of the matter and an infinite time. Another reason for believing the third law is that if one could reduce $\kappa$ to zero by a finite sequence of operations, then presumably one could carry the process further, thereby creating a naked singularity. If this were to happen there would be a breakdown of the assumption of asymptotic predictability which is the basis of many results in black hole theory, including the law that $A$ cannot decrease.

This work was carried out while the authors were attending the 1972 Les Houches Summer School on Black Holes. The authors would like to thank Larry Smarr, Bryce de Witt and other participants of the school for valuable discussions.

\section{References}

1. Hawking, S. W.: Commun. math. Phys. 25, 152--166 (1972).

2. Hawking, S. W.: The event horizon. In: Black Holes. New York, London, Paris: Gordon and Breach 1973 (to be published).

3. Hawking, S. W., Ellis, G.F.R.: The large scale structure of space-time. Cambridge: Cambridge University Press 1973 (to be published).

4. Carter, B.: Phys. Rev. Letters 26, 331-333 (1971).

5. Carter, B.: (Preprint, Institute of Theoretical Astronomy, Cambridge, England).

6. Carter, B.: Properties of the Kerr metric. In: Black Holes. New York, London, Paris: Gordon and Breach 1973 (to be published).

7. Smarr, L.: Phys. Rev. Letters 30, $71-73$ (1973).

8. Beckenstein, J.: PhD Thesis. Princeton University, 1972.

9. Carter, B.: J. Math. Phys. 10, 70-81 (1969).

10. Hawking, S. W.: Commun. math. Phys. 18, 301-306 (1970).

11. Hawking, S. W., Hartle, J. B.: Commun. math. Phys. 27, 283-290 (1972).

12. Bardeen, J. M.: Astrophys. J. 162, $71-95$ (1970).

13. Bardeen, J. M.: Nature 226, 64-65 (1970).

14. Christodoulou, D.: Phys. Rev. Letters 25, 1596-1597 (1970).

J. M. Bardeen

Department of Physics

Yale University

New Haven, Connecticut 06520

USA
B. Carter

S. W. Hawking Institute of Astronomy

University of Cambridge

Cambridge, U.K. 